\title{
ON MALCEV CONDITIONS
}

Dedicated to my mother, in loving memory

WALTER D. NEUMANN

(Received 16 October 1973)

Communicated by M. F. Newman

\section{Introduction}

This note gives a way of looking at Malcev conditions for varieties as ideals in a certain lattice. Though this viewpoint (so far) yields no new results, we feel it puts Walter Taylor's results [3] characterizing "Malcev definable classes of varieties" into a clearer perspective and is therefore worth mentioning. The author is grateful to R. Wille for pointing out W. Taylor's paper to him.

We construct in a simple way a complete lattice $L$ of equivalence classes of varieties containing a countable sublattice $M$ (represented by the finitely presented varieties) such that:

1. equivalent varieties are indistinguishable by Malcev conditions, that is, they either all satisfy a given Malcev condition or all do not;

2. the classes of varieties defined by strong Malcev conditions are just (modulo the equivalence relation) the principal ideals in $L$ generated by single elements of $M$;

3. the classes of varieties defined by ordinary Malcev conditions (respectively, weak Malcev conditions) are the ideals in $L$ generated by subsets of $M$ (respectively, countable intersections of such ideals. Walter Taylor's closure theorems follow easily.

Our lattice $L$ is actually defined on a class, rather than a set. This can be avoided by a cardinality restriction on the number of defining operations that a variety may have; $L$ will then be complete only up to this cardinality.

\section{Construction of $L$}

We first recall the relevant definitions. Let $\mathfrak{M}$ be a finitely presented variety given, say, by the finite set $p_{1}, \cdots, p_{n}$ of primitive operations and a finite set $r_{1}=s_{1}, \cdots, r_{k}=s_{k}$ of equational laws in these operations. An arbitrary variety 
$\mathfrak{B}$ is said to satisfy the strong Malcev condition determined by $\mathfrak{M}$ if there are algebraic operations $\bar{p}_{1}, \cdots, \bar{p}_{n}$ for the variety $\mathfrak{B}$, having the same ranks as the $p_{i}$ and satisfying the given equations. Since then any algebra of $\mathfrak{B}$ is an algebra of $\mathfrak{M}$ with respect to the operations $\bar{p}_{1}, \cdots, \bar{p}_{n}$, we get a functor $\mathfrak{B} \rightarrow \mathfrak{M}$ which preserves underlying sets. Conversely, by Felscher [1], any set preserving functor between varieties arises in the above way, so

Lemma 1. $\mathfrak{B}$ satisfies the strong Malcev condition determined by $\mathfrak{M}$ if and only if there exists a set preserving functor $\mathfrak{B} \rightarrow \mathfrak{M}$.

In particular we see that the condition is independent of the presentation of $\mathfrak{M}$.

Let $\mathscr{V} a r^{f}$ be the category of varieties and set preserving functors. The superscript $f$ stresses that we only allow finitary operations. $\mathscr{V} a r^{f}$ is a complete category (see the appendix for a description of sums and products in this category). For reasons that become clear in the appendix, the terminology "sum and product of varieties" has come to mean categorical sum and product in the dual category $\left(\mathscr{V} a r^{f}\right)^{*}$, that is, product and sum respectively in $\mathscr{V} a r^{f}$. Our notation here therefore differs slightly from [2], where product and sum were taken in the undualized category.

Given $\mathfrak{B}_{1}, \mathfrak{B}_{2} \in \mathscr{V} a r^{f}$, write

$$
\mathfrak{B}_{1} \geqq \mathfrak{B}_{2}
$$

if a morphism $\mathfrak{B}_{1} \rightarrow \mathfrak{B}_{2}$ exists in $\mathscr{V} a r^{f}$. This defines a transitive reflexive relation, so if $\mathscr{V} a r^{\mathcal{f}}$ is factored by the equivalence relation

$$
\mathfrak{B}_{1} \simeq \mathfrak{B}_{2}: \Leftrightarrow \mathfrak{B}_{1} \geqq \mathfrak{B}_{2} \text { and } \mathfrak{B}_{2} \geqq \mathfrak{B}_{1}
$$

we get a partially ordered class

$$
L=\mathscr{F} a r^{\mathcal{L}} / \simeq .
$$

The following lemma is immediate from the categorical definition of sum and product and the fact that sum and product of two finitely presented varieties are still finitely presented.

LEMMA 2. L is a complete lattice with meet and join given by product and sum of varieties (that is, in $\left.\left(\mathscr{V} a r^{f}\right)^{*}\right)$. The subset $M$ of elements of $L$ represented by finitely presented varieties is a countable (not complete) sublattice of $L$.

Now if $\mathfrak{M}$ is a finitely presented variety, then by lemma 1 a variety $\mathfrak{B}$ satisfies the corresponding strong Malcev condition if and only if $\mathfrak{B} \geqq \mathfrak{M}$. Two varieties $\mathfrak{B}_{1}, \mathfrak{B}_{2}$ which define the same element of $L$ (that is $\mathfrak{B}_{1} \cong \mathfrak{B}_{2}$ ) either both satisfy such a condition or both do not, so such varieties will be called Malcev indistinguishable, and in discussing classes of varieties defined by Malcev conditions we lose nothing by working modulo this relation; that is, we work in $L$ rather than in $\mathscr{V} a r^{S}$. 
The following proposition is trivial:

Proposition 1. A subclass of $L$ is defined by a strong Malcev condition if and only if it is a principal ideal in $L$ generated by an element of $M$ (namely the element given by a variety which defines the strong Malcev condition in question).

Now an ordinary Malcev condition is defined as a countably infinite logical alternation

$$
C=C_{1} \vee C_{2} \vee \cdots \quad\left(C_{1} \Rightarrow C_{2} \Rightarrow \cdots\right)
$$

of a chain of strong Malcev conditions.

Proposition 2. A subclass of Lis defined by a Malcev condition if and only if it is an ideal in $L$ generated by a subset of $M$.

PROOF. For each $i$ let $\mathfrak{M}_{i}$ be a finitely presented variety which determines the strong Malcev condition $C_{i}$ and let $K_{i}$ be the principal ideal in $L$ generated by $\left[\mathfrak{M}_{i}\right]$. The subclass of $L$ determined by condition $C$ is

$$
K=K_{1} \cup K_{2} \cup \cdots,
$$

which is itself an ideal since $K_{1} \subseteq K_{2} \subseteq \cdots$, and is generated by the subset $\left\{\left[\mathfrak{M}_{1}\right],\left[\mathfrak{M}_{2}\right], \cdots\right\}$ of $M$.

Conversely, let $K$ be an ideal in $L$ generated by a subset $\left\{\left[\mathfrak{M}_{1}^{\prime}\right],\left[\mathfrak{M}_{2}^{\prime}\right], \cdots\right\}$ of $M$ (we can index this way since $M$ is countable). Then $K$ is also generated by $\left\{\left[\mathfrak{M}_{1}\right],\left[\mathfrak{M}_{2}\right], \cdots\right\}$, where

$$
\left[\mathfrak{M}_{i}\right]=\left[\mathfrak{M}_{1}^{\prime}\right] \wedge\left[\mathfrak{M}_{2}^{\prime}\right] \wedge \cdots \wedge\left[\mathfrak{M}_{i}^{\prime}\right]=\left[\mathfrak{M}_{1}^{\prime}+\cdots+\mathfrak{M}_{i}^{\prime}\right] .
$$

Here $\wedge$ is the lattice operation in $M$, given by the categorical sum + in $\left(\mathscr{V} a r^{\top}\right)^{*}$ (lemma 1). Since $\mathfrak{M}_{1} \geqq \mathfrak{M}_{2} \geqq \cdots$, we have for the corresponding principal ideals $K_{i}$ in $L$ that $K_{1} \subseteq K_{2} \subseteq \cdots$. Hence $K$ is the union $K_{1} \cup K_{2} \cup \cdots$ and is determined by the Malcev condition as above.

Before discussing weak Malcev conditions we need a lemma.

LEMma 3. The logical conjunction of a finite number of Malcev conditions is a Malcev condition. Equivalently, the intersection of a finite number of ideals of the type of proposition 2 again has this type.

Proof. We need only prove this for the intersection of two such ideals $K$ and $K^{\prime}$. But if $K$ is generated by the elements $\left[\mathfrak{M}_{i}\right] \in M$ and $K^{\prime}$ by the elements $\left[\mathfrak{M}_{j}^{\prime}\right] \in M$, then $K \cap K^{\prime}$ is generated by the elements $\left[\mathfrak{M}_{i}\right] \vee\left[\mathfrak{M}_{j}^{\prime}\right] \in M$.

Finally we come to weak Malcev conditions. These are logical conjunctions 


$$
C=C_{1} \wedge C_{2} \wedge \cdots
$$

of a chain

$$
C_{1} \Leftarrow C_{2} \Leftarrow \cdots
$$

of progressively stronger Malcev conditions.

However, given any countable logical conjunction

$$
C=C_{1}^{\prime} \wedge C_{2}^{\prime} \wedge \cdots
$$

of Malcev conditions, we can write it as

$$
C=C_{1} \wedge C_{2} \wedge \cdots, \quad\left(C_{1} \Leftarrow C_{2} \Leftarrow \cdots\right),
$$

where $C_{i}=C_{1}^{\prime} \wedge \cdots \wedge C_{i}^{\prime}$, which is a Malvec condition by the above lemma. Thus any countable conjunction of Malcev conditions is a weak Malcev condition (and vice versa), so

Proposition 3. A subclass of $L$ is defined by a weak Malcev condition if and only if it is a countable intersection of ideals, each of which is generated by a subset of $M$.

\section{Closure theorems}

As example applications of the viewpoint developed above, we derive two of W. Taylor's closure theorems [3]. We must first recall a definition ([2] p. 6): a set preserving functor $F: \mathfrak{B}_{1} \rightarrow \mathfrak{B}_{2}$ between varieties is a pure forgetful functor if $\mathfrak{B}_{2}$ is generated as a variety by the image of $F$, that is, $\mathfrak{V}_{2}=Q \operatorname{SP}\left(F \mathfrak{B}_{1}\right)$.

We now formulate W. Taylor's theorem 4.1, giving in round brackets the formulation in our language.

THEOREM. A non-empty class $K$ of varieties is definable by a [strong] Malcev condition if and only if it satisfies the following five conditions:

(i) $K$ is closed under formation of equivalent varieties;

(ii) $K$ is closed under formation of subvarieties;

(iii) $K$ is closed under formation of [countable] respectively finite products of varieties;

(iv) if $\mathfrak{B} \in K$ and $\mathfrak{B}$ is generated by all reducts of members of $\mathfrak{W}$ to the type of of $\mathfrak{V}$ then $\mathfrak{W} \in K$ (if $\mathfrak{B} \in K$ and $\mathfrak{W} \rightarrow \mathfrak{B}$ is a pure forgetful functor then $\mathfrak{W} \in K$ );

(v) if the equations $\Sigma$ define a variety on $K$ of type $\tau$, then there exist finite subsets $\Sigma_{0} \subseteq \Sigma, \tau_{0} \subseteq \tau$, such that $\Sigma_{0}$ defines a variety in $K$ of type $\tau_{0}$ (if $\mathfrak{B} \in K$ then there exists a finitely presented variety $\mathfrak{M} \in K$ and a set preserving functor $\mathfrak{B} \rightarrow \mathfrak{M})$.

The equivalence of formulations is immediate from the fact that set preserving functors are equationally defined and vice versa. 
Proof of TheOREM. Since trivially any morphism (set preserving functor) $F: \mathfrak{V}_{1} \rightarrow \mathfrak{B}_{2}$ of varieties splits (uniquely) as a pure forgetful functor

$$
\mathfrak{B}_{1} \rightarrow \operatorname{QSP}\left(F \mathfrak{V}_{1}\right)
$$

followed by an inclusion $Q S P\left(F \mathfrak{B}_{1}\right) \rightarrow \mathfrak{B}_{2}$, conditions (i), (ii), and (iv) just say that if $\mathfrak{B}_{1} \rightarrow \mathfrak{B}_{2}$ and $\mathfrak{B}_{2} \in K$, then $\mathfrak{B}_{1} \in K$. That is, $K$ contains with any variety also all larger varieties (in our order relation $\geqq$ ) and is hence in particular closed under "Malcev indistinguishability". We thus lose nothing by working modulo Malcev indistinguishability, that is in $L$, and considering $K$ as a subclass of $L$. Since product of varieties is meet in our lattice $L$, adding condition (iii) to (i), (ii), and (iv) just says $K$ defines an ideal in $L$. Condition (v) then says every element of $K$ is greater than an element of $M \cap K$, so $K$ is generated by the subset $M \cap K$ of $M$ and is thus defined by a Malcev condition by proposition 2. If $K$ is actually closed under countable meets (condition (iii) [3]) then, since $M \cap K$ is countable, $K$ is the principal ideal generated by the meet of the elements in $M \cap K$. This smallest element of $K$ must be in $M$ by condition (v), so $K$ is defined by a strong Malcev condition. The converse is trivial in both cases.

The second closure theorem is as follows (Theorem 4.2 of [3]):

TheOREM. A nonempty class $K$ of varieties is definable by a [strong] Malcev condition if and only if $K$ satisfies conditions (i), (ii), (ii) of the previous theorem and

(vi) if the equations $\Sigma$ define a variety $\mathfrak{B}$ in $K$ of type $\tau$, then there exists a finite subset $\Sigma_{0} \subset \Sigma$ defining a variety in $K$ of type $\tau$.

ProOF. Let us first investigate what (vi) means in our language. Let us first assume the type $\tau$ has $\mathfrak{n}$ operations of each finite rank, where $\mathfrak{n}$ is some large infinite cardinal. This can be achieved without changing the variety $\mathfrak{B}$ (up to equivalence) by simply adding operations and simultaneously adding equations which set the new operations equal to old operations of $\mathfrak{P}$. The finite set $\Sigma_{0}$ of equations can only involve a finite subset $\tau_{0} \subset \tau$. So the variety of type $\tau$ defined by $\Sigma_{0}$ splits as a sum of varieties

$$
\mathfrak{M}+\mathfrak{F}
$$

$\mathfrak{M}$ of type $\tau_{0}$ defined by $\Sigma_{0}$, and $\mathfrak{F}$ of type $\sigma=\tau-\tau_{0}$ with no equations. We thus have the weaker version of (vi):

(vii) if $\mathrm{n}$ is an infinite cardinal, $\mathfrak{F}$ the variety with $\mathrm{n}$ operations of each rank and with no laws, then for any $\mathfrak{B} \in K$ there exists for $n$ sufficiently large a finitely presented variety $\mathfrak{M}$ such that $\mathfrak{M}+\mathfrak{F} \in K$ and $\mathfrak{B} \subseteq \mathfrak{M}+\mathfrak{F}$.

Now suppose $\mathfrak{B} \in K$ and $\mathfrak{W} \rightarrow \mathfrak{B}$ is any set preserving functor. We wish to show $\mathfrak{W} \in K$. By choosing $\mathfrak{n}$ large enough we can embed $\mathfrak{W}$ in $\mathfrak{F}$ as a subvariety:

$$
\mathfrak{W} \subseteq \mathfrak{\&}
$$


Let

$$
\mathfrak{B} \subseteq \mathfrak{M}+\mathfrak{F}
$$

be given by (vii). Thirdly, since $n$ is infinite, we have an equivalence of varieties

$$
\mathfrak{F}+\mathfrak{F} \equiv \mathfrak{F}
$$

Now the functor $\mathfrak{W} \rightarrow \mathfrak{B}$ yields an inclusion of varieties (the diagonal map) $\mathfrak{W} \subseteq \mathfrak{B}+\mathfrak{B}$, so using the above inclusion and equivalence we get

$$
\mathfrak{B} \subseteq \mathfrak{B}+\mathfrak{W} \subseteq \mathfrak{B}+\mathfrak{F} \subseteq(\mathfrak{M}+\mathfrak{F})+\mathfrak{F} \equiv \mathfrak{M}+\mathfrak{F} .
$$

This gives $\mathfrak{B}$ as a subvariety of a variety in $K$, hence $\mathfrak{B}$ is in $K$ by (ii).

Thus $K$ contains with any variety also all larger (with respect to $\geqq$ ) varieties. As before, $K$ is thus closed under Malcev indistinguishability and can thus be considered as a subclass of $L$. It is then an ideal in $L$ by condition (iii) (product closure in $\left(\mathscr{V} a r^{f}\right)^{*}$, that is, closure under meets in $\left.L\right)$.

In condition (vii) we see that $\mathfrak{M}$ and $\mathfrak{M}+\mathfrak{F}$ are Malcev indistinguishable (since set preserving functors $\mathfrak{M} \rightarrow \mathfrak{M}+\mathfrak{F}$ and $\mathfrak{M}+\mathfrak{F} \rightarrow \mathfrak{M}$ certainly exist), so (vii) says that each element of $K$ is greater than some element of $M \cap K$, so, as before, $K$ is generated by $M \cap K$ [respectively, the meet of all elements of $M \cap K$ ].

We now give the description of Walter Taylor's heirarchy [3] of subclasses of $\mathscr{V} a r^{f}$ in our language. The proofs of the equivalence of our description in cases 4 and 5 are exactly like the proofs of the above two theorems and can be filled in by the reader.

Denote by $M_{1}$ the set of elements in $L$ which are joins of elements in $M$, that is, are representable by sums of finitely presented varieties. These joins and sums can be assumed to be countable since $M$ is countable. Let $M_{2}$ be the set of elements in $L$ representable by countably presented varieties. Clearly $M_{1} \subseteq M_{2}$ Taylor's hierarchy is:

1). Classes defined by strong Malcev conditions, i.e. principal ideals in $L$ generated by an element of $M$.

2). Classes defined by Malcev conditions, i.e. ideals in $L$ generated by subsets of $M$.

3). Classes defined by weak Malcev conditions, i.e. countable intersections of ideals generated by subsets of $M$.

4). Ideals $K$ in $L$ generated by subsets of $M_{1}$ and satisfying $\left({ }^{*}\right)$ : a meet of a family of elements of $L$ is in $K$ if the meet of every countable subfamily is in $K$.

5). Ideals in $L$ generated by subsets of $M_{2}$.

This description suggests several additions one could make to this heirarchy. Most interesting seems to be the following addition between 3) and 4):

$3 \frac{1}{2}$ ). Arbitrary intersections of ideals generated by subsets of $M$. 
That hierarchy 3) is contained in hierarchy $3 \frac{1}{2}$ ) is trivial. Now suppose a class $K$ is of hierarchy $3 \frac{1}{2}$ ), that is, $K$ is an intersection of ideals of hierarchy 2). Since each ideal of hierarchy 2 ) satisfies condition $\left({ }^{*}\right)$, their intersection $K$ also does. But $K$ is certainly generated by a set of the joins of elements of $M$, that is, by a subset of $M_{1}$, so $K$ is of hierarchy 4 ).

The above makes it very unlikely that hierarchies 3 ) and 4) coincide. In fact, it seems probable that $3 \frac{1}{2}$ ) lies strictly between 3 ) and 4 ). The non-equality of 3 ) and $3 \frac{1}{2}$ ) would imply that there are uncountably many different classes defined by Malcev conditions (W. Taylor's problem 4.6), which seems not yet be known.

\section{Appendix: Sum and product of varieties}

The category $\mathscr{V} a r^{f}$ is a full subcategory of the category $\mathscr{V} a r$ (see [2]) of varieties allowing $n$-ary operations also for $n$ countably infinite. Recall that sum and product of varieties is used to mean categorical sum and product in the dual category $\left(\mathscr{V} a r^{\mathcal{S}}\right)^{*}$, which is a full subcategory of $(\mathscr{V} a r)^{*}$. The description of sum and product in $(\mathscr{V} a r)^{*}$ (see [2]) can easily be transferred to $\left(\mathscr{V} a r^{f}\right)^{*}$ as follows:

Given varieties $\mathfrak{B}_{i}(i \in I)$, each $\mathfrak{B}_{i}$ defined by a set $F_{i}$ of operations and a set $\Sigma_{i}$ of equational laws in these operations, the sum $\mathfrak{B}$ in $(\mathscr{V} \text { ar })^{*}$ of the $\mathfrak{B}_{i}$ is defined by taking the disjoint union of the sets $F_{i}$ as set of operations and the disjoint union of the $\Sigma_{i}$ as the equations ([2]). Since, if the $\mathfrak{B}_{i}$ are finitary then so is $\mathfrak{B}$, this also describes sum in $\left(\mathscr{V} a r^{\mathfrak{J}}\right)^{*}$.

Product causes trouble because an infinite $(\mathscr{V} \text { ar })^{*}$-product of finitary varieties is no longer finitary. However, it is easily seen directly that the $\left(\mathscr{V} a r^{f}\right)^{*}$ product is just the reduct of the $(\mathscr{V} a r)^{*}$-product to the set of all its finitary opera-

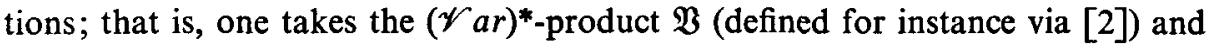
then if $F$ is the set of all finitary algebraic operations of $\mathfrak{B}$, and $\Sigma$ the set of all equations defined over $F$ and valid in $\mathfrak{B}$, then $F$ and $\Sigma$ together define the $\left(\mathscr{V} a r^{f}\right)^{*}$-product.

This can also be proved using $\aleph_{0}$-clones [2]. Recall that the variety of $\aleph_{0}$ clones is defined by one operation $(\cdots)$ of rank $\omega$ and a countable sequence of nullary operations (constants) $p_{0}, p_{1}, \cdots$ satisfying the laws

C1) $\left(x p_{1} p_{2} \cdots\right)=x$

C2) $\left(p_{i} x_{1} x_{2} \cdots\right)=x_{i} \quad(i=1,2, \cdots)$

C3) $\left(\left(x y_{1} y_{2} \cdots\right) z_{1} z_{2} \cdots\right)=\left(x\left(y_{1} z_{1} z_{2} \cdots\right)\left(y_{2} z_{1} z_{2} \cdots\right) \cdots\right)$.

By [2], the variety $\mathfrak{C}$ of $\aleph_{0}$-clones is equivalent as a category to $(\mathscr{V} a r)^{*}$. This equivalence

$$
H:(\mathscr{V} a r)^{*} \rightarrow \mathbb{C}
$$

is defined by taking $H(\mathfrak{B})$ to be the set of $\omega$-ary algebraic operations of $\mathfrak{B}$ and defining $p_{i}$ to be the $i$-th coordinate operation and 


$$
\left(f f_{1} f_{2} \cdots\right)=f\left(f_{1}, f_{2}, \cdots\right)
$$

(right hand side is composition of operations).

Now by [2], $\left(\mathscr{V} a r^{f}\right)^{*}$ gets mapped to the subclass $\mathfrak{C}^{f}$ of $\mathfrak{C}$ consisting of all $\aleph_{0}$-clones which satisfy

$$
\exists_{n}\left(x p_{1} p_{2} \cdots p_{n} p_{1} p_{1} \cdots\right)=\left(x p_{1} p_{2} \cdots p_{n} p_{n+1} \cdots\right),
$$

for every element $x$. In an arbitrary $\aleph_{0}$-clone $H$, the set of elements $x$ satisfying $F$ ) is a sub-clone $H^{f}$. It follows immediately that for a family of $\aleph_{0}$-clones $H_{i} \in \mathbb{C}^{f}$, the $\mathfrak{C}^{\mathcal{f}}$-product is just $\left(\times C_{i}\right)^{\mathcal{f}}$, where $\times C_{i}$ is cartesian product (i.e. product in $\mathfrak{C}$ ). Our claim about product in $\left(\mathscr{V} a r^{f}\right)^{*}$ follows then from the relationship between $(\mathscr{V} a r)^{*}$ and $\mathfrak{C}$.

For two varieties $\mathfrak{B}_{1}$ (defined by a set $F$ of operations and a set $\Sigma_{1}$ of equational laws) and $\mathfrak{B}_{2}$ (defined by operations $G$ and laws $\Sigma_{2}$ ), the product $\mathfrak{B}_{1} \times \mathfrak{B}_{2}$ can be defined by the disjoint union $F \cup G \cup\{\cdot\}$ of operations, where $\cdot$ is a binary product, and the disjoint union $\Sigma_{1} \cup \Sigma_{2} \cup \Sigma$ of laws, where $\Sigma$ is the set of laws $(f \in F, g \in G)$ :

$$
\begin{aligned}
x \cdot x & =x & & \\
(x \cdot y) \cdot z & =x \cdot(y \cdot z)=x \cdot z & & \\
f\left(x_{1}, \cdots, x_{i} \cdot y_{i}, \cdots, x_{k}\right) & =f\left(x_{1}, \cdots, x_{k}\right) & & \\
& =f\left(x_{1}, \cdots, x_{k}\right) \cdot y_{1} & & , i>1 \\
g\left(y_{1}, \cdots, x_{i} \cdot y_{i}, \cdots, y_{l}\right) & =g\left(y_{1}, \cdots, y_{l}\right) & & , i=1 \\
& =x_{1} \cdot g\left(y_{1}, \cdots, y_{l}\right) & & , i>1 \\
f\left(x_{1}, \cdots, x_{i-1}, g\left(y_{1}, \cdots, y_{l}\right), \cdots, x_{k}\right) & =f\left(x_{1}, \cdots, x_{i-1}, y_{1}, \cdots, x_{k}\right) & & , i=1 \\
& =f\left(y_{1}, x_{2}, \cdots, x_{k}\right) \cdot g\left(y_{1}, \cdots, y_{l}\right) & & , i=1 \\
g\left(y_{1}, \cdots, y_{i-1}, f\left(x_{1}, \cdots, x_{k}\right), \cdots, y_{l}\right) & =g\left(y_{1}, \cdots, y_{i-1}, x_{1}, \cdots, y_{l}\right) & & , i>1 \\
& =f\left(x_{1}, \cdots, x_{k}\right) \cdot g\left(x_{1}, y_{2}, \cdots, y_{l}\right) & & , i=1
\end{aligned}
$$

This is maybe most easily seen by observing that the $\aleph_{0}$-clone of the variety $\mathfrak{B}$ defined by $F \cup B \cup\{\cdot\}$ and the laws $\Sigma_{1} \cup \Sigma_{2} \cup \Sigma$ is in an obvious way naturally isomorphic as a set to the cartesian product of the $\aleph_{0}$-clones of $\mathfrak{V}_{1}$ and $\mathfrak{B}_{2}$ (namely, first extend all operations trivially to have rank $\omega$; then using the equations, every rank $\omega$ operation of $V$ has form $q_{1} \cdot q_{2}$ where $q_{1}$ is a term in the operations $F$ and the coordinate operations, and $q_{2}$ in the operations $G$ and the coordinate operations; map this to the pair $\left.\left(q_{1}, q_{2}\right) \in H\left(\mathfrak{B}_{1}\right) \times H\left(\mathfrak{B}_{2}\right)\right)$. Check that this is an $\aleph_{0}$-clone isomorphism. 
The above descriptions are probably well known; we have included them for completeness. They show in particular that finite sum and product of finitely presented varieties are finitely presented, a fact used in this paper.

\section{References}

[1] W. Felscher, 'Equational maps', Contrib. to Math. Logic, (Amst erdam 1968), 121-161.

[2] W. D. Neumann, 'Representing varieties of algebras by algebras', J. Aust. Math. Soc. 11 (1970), 1-8.

[3] W. Taylor, 'Characterizing Mal'cev conditions', Preprint.

Mathematisches Institut

Universität Bonn

Germany 